\title{
Agricultural Education through Farm Tourism Among Selected Providers in Mindanao
}

\author{
Fernando V. Magdato, Jr ${ }^{a}$. and Rowena DT. Baconguis ${ }^{b}$ \\ fvmagdatojr@gmail.com \\ ${ }^{a}$ Associate Professor, CARS - USeP Apokon, Tagum City 8100, Davao del Norte Philippines \\ ${ }^{b}$ Professor, CPAf - UP Los Banos, Laguna 4031, Laguna Philippines
}

\begin{abstract}
Farm tourism is a new economic development strategy in the Philippines which helps uplift the living and working conditions of farmers by generating additional farm-related income that enhance farm profitability at the same time that it provides agricultural education to various stakeholders. Case studies of selected farm tourism sites in Mindanao, Philippines were conducted using key informant interviews and use of GIS mapping. Farm tourism sites were characterized based on agricultural technologies, agricultural produce and tourism activities offered in the area. The study examined what agricultural knowledge was shared in these farm tourism sites, how it was shared and who the major clients were. Results revealed that the development of farm tourism sites is influenced by the philosophy of the farm operators who believe that farming is a profitable business, an opportunity to promote common good and love for agriculture. Knowledge sharing activities center on the conduct of training programs, farm tours to demonstration sites, recreational activities where visitors engage in harvesting and fishing. Knowledge sharing activities are mostly conducted through group and interpersonal approaches and mass media approach through the dissemination of agricultural technology which is central to all farm tourism operators.
\end{abstract}

Published by IJRP.ORG. Selection and/or peer-review under responsibility of International Journal of Research Publications (IJRP.ORG)

Keywords: Agricultural Education; Farm Tourism; Philosophy of Farm Tourism; Knowledge Sharing

\section{Introduction}

\subsection{Rationale}

Farmers in developing nations like the Philippines are experiencing tremendous challenges due to several factors such as globalization, free-trade, falling commodity prices, oversupply of goods, changes in subsidy policies and increased reliance on technologically intensive production (Che, Veeck, \& Veeck, 2005; Phelan $\&$ Sharpley, 2011). In addition, McGehee et al. (2007) recognized that the agricultural industry is suffering from a variety of problems including environmental pressures, climate change, decline in terms of trade in agriculture, low-income elasticities in commodity markets, and over reliance on raw products. This inevitably makes diversification of farming activities a must. This realization rallied the traditional agriculture sector towards developing farm tourism which is expected to lead economic growth within a rural setting.

A number of agricultural producers have engaged in the agri-tourism industry by opening their farms to visitors, travellers and families seeking recreational or educational activities. The growing consumer demands for organic food and desire to combine potentials of farm tourism led to the development of farm tourism. In House Bill No. 1808, agritourism or farm tourism refers to a working farm ranch or any agricultural operation opened for public visitation for their enjoyment, outdoor recreation activities, education, shopping, dining or even lodging. Farm tourism primarily developed to generate additional income to the farmer and for its economic benefits to the local economy (Inskeep, 1991); Elson, Steenberg and Wilkinson (1995). Literature 
suggests that farm tourism help mitigate the negative environmental effects from intensive agriculture such as soil erosion, water pollution and stream bank erosion, increase landscape beautification, provide barriers to residential sprawl and encourage conservation of native habitats and wildlife among others (Gold, Cernusca, \& Godsey, 2009; Lambert, Sullivan, Claassen, \& Foreman, 2006).

Incorporating tourism related activities may have started hundreds of years ago in Northern America and intensified in the 1980's as a strategy to increase farm income by offering a variety of products and activities (Rendle, 2000). Different countries now promote the incorporation of tourism as a strategy to ensure sustainability of family farms, such as Italy (Chase et al., 2018), Austria (Rendle, 2000), Poland, (BajgierKowalska, Tracz\& Uliszak, 2017) Nepal (Pande \& Pandey) Malaysia, (Tiraieyari and Hamzah , 2012), Indonesia (Kurnianto, B. T., Sugiyanto, Hidayat, K., \& Sukesi, K.2013). and Thailand (Choenkwan et al., 2016)

Realizing the importance of the tourism industry to the national economy, the Philippine government has taken various initiatives to further develop it, and has put particular emphasis on the development of the industry through the Department of Tourism (DOT) and Department of Agriculture-Agricultural Training Institute (DA-ATI). There are various programs conducted by the government to further promote agri-tourism activities. For instance, the "agriculture and tourism", widely considered as a powerful driver of economic growth, is one of the key priority areas of the Philippine Development Plan 2011-2016, which was formulated to further develop agro-tourism or the home-stay industry as a key driver of rural community development. In addition, the Aquino government believes in farm tourism for the Philippines. The President is promoting further development of farm tourism nationwide, believing farm tourism will help boost better socioeconomic development in the country. Government's bid to promote farm tourism as one of the key mechanisms will ensure the success of community development has gone full swing.

There are few recognized farm tourism sites in the Philippines. From 2009-2010 Mindanao is among the top five tourist destinations of the country in terms of number of domestic and foreign visitors (DOT, 2010). Perhaps one of the top farm tourism destinations in the country is situated in Mindanao, particularly Mindanao Baptist Rural Life Center (MBRLC), a non-government organization based in Kinuskusan, Bansalan, Davao del Sur. People from all over the world, from Australia to Zimbabwe, have come to see the area. Most of its visitors, however, are interested to see and learn the "sustainable farming systems" which the center has developed through the years of experimentation and consultations. Every year, MBRLC is host to almost 10,000 annual visitors (http://blog.agriculture.ph/category/agri-tourism).

Being a young industry, farm tourism in Mindanao is still growing and developing. Thus, there is limited information regarding the current agricultural knowledge sharing within the context of farm tourism. However because of potential benefits of educating a diverse group of stakeholders through farm tourism, there is a need to investigate the agricultural knowledge sharing in a farm tourism set up. In doing so, the paper seeks to contribute to improving the potentials of farm tourism in capacitating groups about farm knowledge and specific technologies.

\subsection{Objectives of the Study}

The main objective of the study was to examine what agricultural knowledge was shared in these farm tourism sites, how it was shared and who the major clients were.

\subsection{Importance of the Study}

According to Lobo et al. (1999) the growth of the farm tourism industry requires the coordination of institutions and organizations that can coordinate strategic planning, promotion, and information management. The educational value of farm tourism could create awareness about rural life and knowledge about agriculture science among future agricultural leaders and practitioners. It provides an alternative for school 
picnics and opens up opportunity for hands-on farm management experience for urban college students in agriculture. It can be effectively used as an educational and training tool to equip line department officers, retirees, returning overseas workers and other groups of individuals interested in farm tourism management. It serves as a technology demonstration in showcasing good agricultural practices, processes and technologies. In short, the operation of farm tourism provides a unique opportunity for education through recreation where learning is targeted to be fun, effective and easy.

\subsection{Scope and Limitations of the Study}

While there are many important aspects to investigate, the scope of the study was limited to agricultural knowledge sharing in farm tourism. It covered extension aspects among selected major farm tourism operators in Mindanao engaged in farming production activities, non-formal agricultural education and tourism operation.

The six (6) selected farm tourism sites considered for the study were Mindanao Baptist Rural Life Center (Bansalan, Davao del Sur), Lao Integrated Farm, Inc. (Eman, Bansalan, Davao del Sur), ACES Natural Farm (Peda, San Francisco, Panabo City), Paradise Organic Farm (Koronadal City, South Cotabato), Binahon Farm (Songco, Lantapan, Bukidnon), and Dumandan Greenfields Diversified Farm (Baguio, Calinan Davao City).

The respondents included only the owners and managers of the identified six (6) farm tourism sites in Mindanao, Philippines. The visitors were not interviewed in this study.

\section{Methodology}

This study employed the qualitative study research design. Data were gathered using key informant interviews, field observations, informal discussions, personal communications, and community documents. The research participants were the farm tourism owner-operators.

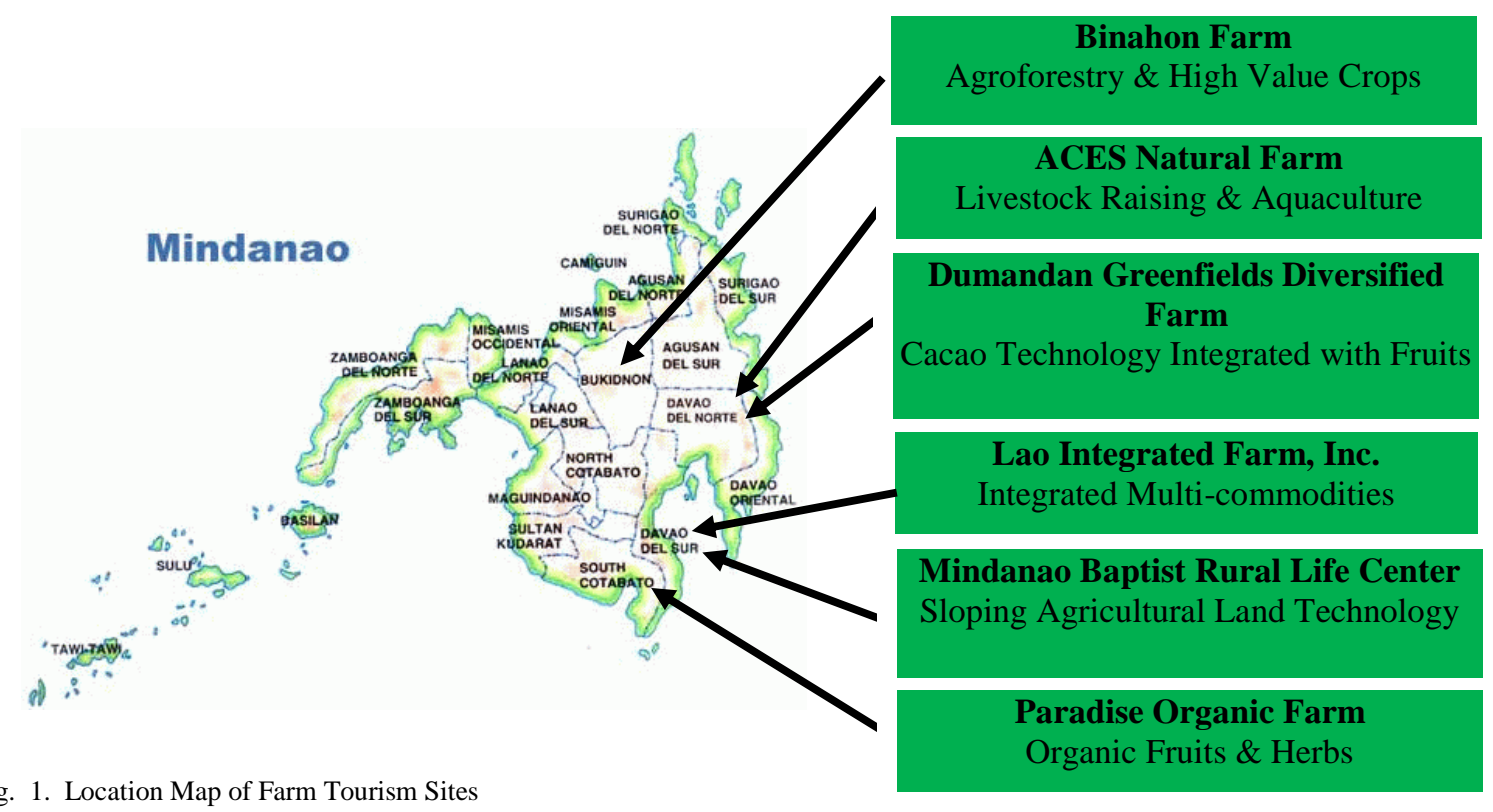

Fig. 1. Location Map of Farm Tourism Sites

This study focused on the agricultural knowledge sharing in farm tourism and also covered extension aspects among selected major farm tourism operators in Mindanao engage in farming production activities 
and in managing their farms. The key farmer participants were chosen with the help of Agricultural Training Institute (ATI) and Southern Mindanao Agriculture and Resources Research and Development Consortium (SMARRDEC). The selection was based on the following criteria: The farm shall be in operation for more than 5 years and frequently visited by tourist. The identified farm tourism provider in Mindanao is shown in Figure 1 below.

Binahon Farm, was chosen based on the Agroforestry technology promoted to soil and conservation farming system among farmers in Bukidnon. ACES Natural Farm, on the other hand, was chosen based on the naturally grown technology promoted to milkfish, native chicken, hog, vegetables and feeds farmers in Davao del Norte. Dumandan Greenfields Diversified practiced diversified farming systems and was chosen based on the cacao technology integrated with durian, lanzones (Longkong), coconut and arabica coffee promoted to farmers of Davao City. While Lao Integrated Farm, Inc. was chosen based on the coco-sugar and syrup technologies promoted to coconut farmers in Davao del Sur. Furthermore, Mindanao Baptist Rural Life Center (MBRLC) was chosen based on the soil conservation technology promoted and because of the notable number of Sloping Agricultural Land Technology (SALT) users in Davao del Sur. Paradise Organic Farm was chosen based on the technology on organic diversified fruit trees, promotion to fruit tree farmers of Koronadal, South Cotabato. Paradise Organic Farm also produces all- organic fresh fruits, herbal medicines and supplements, organic fertilizer, OPV vegetable seeds, organic vegetable and rice. At Paradise Organic Farm they sell organic fresh fruits, herbal medicines \& supplements, organic fertilizer, OPV vegetable seeds, organic vegetable \& rice.

\subsection{Conceptual Framework}

This study used the conceptual framework shown in Figure 2 below.

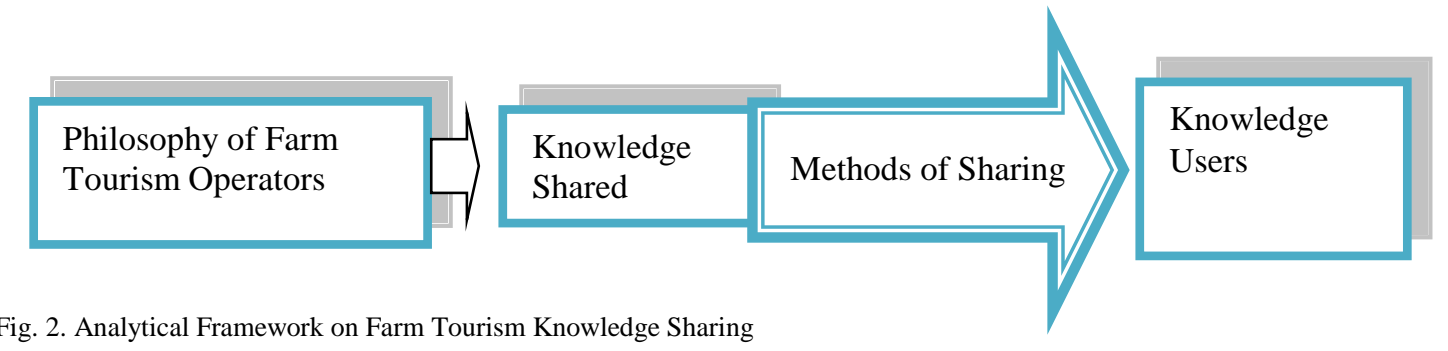

The analytical framework takes off from the knowledge management theory which emphasizes sharing as an important aspect of learning. Sharing involves communication or "the exchange of messages" which leads to "commonness" between two or more parties through a particular medium, or an active, dynamic process in which ideas and information are exchanged leading to modification of people's knowledge, attitudes and practices (Burnett, 2003). Thus, methods of sharing is investigated. Moreover, the analytical framework presupposes that the kinds of technologies shared is influenced primarily by the philosophy of the farm operator and the kinds of support services accessed which help the farm operator developed their technologies. Finally, the analytical framework investigates the learners who seek knowledge in a farm tourism modality.

\section{Results of the Study}

Farm tourism research sites are usually big in land area and practice diversified farming with specialized technology. ACES Natural Farm was chosen based on the naturally grown technology promoted to milkfish, native chicken, hog, vegetables and feeds farmers in Davao del Norte. Binahon Farm, on the other hand, was 
chosen based on the Agroforestry technology promoted to soil and conservation farming system among farmers in Bukidnon. While Lao Integrated Farm, Inc. was chosen based on the coco-sugar and syrup technologies promoted to coconut farmers in Davao del Sur. Furthermore, Mindanao Baptist Rural Life Center (MBRLC) was chosen based on the soil conservation technology promoted and because of the notable number of Sloping Agricultural Land Technology (SALT) users in Davao del Sur. Paradise Organic Farm was chosen based on the technology on organic diversified fruit trees, promotion to fruit tree farmers of Koronadal, South Cotabato. Paradise Organic Farm also produces all- organic fresh fruits, herbal medicines and supplements, organic fertilizer, OPV vegetable seeds, organic vegetable and rice. Dumandan Greenfields Diversified Farm was chosen based on the cacao technology integrated with durian, lanzones (Longkong), coconut and Arabica coffee promoted to farmers of Davao City.

Table 1. Characteristics of the farm

\begin{tabular}{|c|c|c|c|c|c|c|c|c|}
\hline \multirow{2}{*}{$\begin{array}{c}\text { Farm } \\
\text { tourism } \\
\text { operator }\end{array}$} & \multirow{2}{*}{$\begin{array}{l}\text { Size } \\
\text { (ha) }\end{array}$} & \multirow{2}{*}{$\begin{array}{l}\text { Agricultural } \\
\text { technologies }\end{array}$} & \multicolumn{6}{|c|}{ Agricultural produce } \\
\hline & & & Livestock & $\begin{array}{l}\text { fish, } \\
\text { others }\end{array}$ & $\begin{array}{l}\text { vegetable, } \\
\text { field crops }\end{array}$ & $\begin{array}{l}\text { fruits, } \\
\text { plantation } \\
\text { crops }\end{array}$ & inputs & $\begin{array}{l}\text { Processed } \\
\text { foods }\end{array}$ \\
\hline $\begin{array}{l}\text { Binahon } \\
\text { Farm }\end{array}$ & 8 & $\begin{array}{l}\text { Agro-forestry, } \\
\text { Sloping } \\
\text { Agricultural } \\
\text { Land } \\
\text { Technology } \\
\text { (SALT), } \\
\text { organic } \\
\text { farming }\end{array}$ & $\begin{array}{l}\text { Carabao, } \\
\text { horse, goat, } \\
\text { sheep, hogs, }\end{array}$ & $\begin{array}{l}\text { Tilapia, } \\
\text { exotic } \\
\text { honey } \\
\text { bees }\end{array}$ & $\begin{array}{l}\text { Cabbage, } \\
\text { carrots, } \\
\text { cauliflower, } \\
\text { lettuce, } \\
\text { tomatoes, } \\
\text { potato, green } \\
\text { onions }\end{array}$ & Lanzones & $\begin{array}{l}\text { Organic } \\
\text { fertilizer }\end{array}$ & $\begin{array}{l}\text { Carrot calamansi } \\
\text { juice, vegetable } \\
\text { pickles }\end{array}$ \\
\hline $\begin{array}{l}\text { ACES } \\
\text { Natural } \\
\text { Farm }\end{array}$ & 4 & $\begin{array}{l}\text { Diversified } \\
\text { farming } \\
\text { systems, } \\
\text { Organic } \\
\text { farming }\end{array}$ & $\begin{array}{l}\text { Hog, } \\
\text { chicken }\end{array}$ & Milkfish & $\begin{array}{l}\text { Vegetables: } \\
\text { tomato, egg } \\
\text { plant, pechay, } \\
\text { squash, lettuce }\end{array}$ & $\begin{array}{l}\text { Cacao, coffee } \\
\text { lanzones } \\
\text { coconut, } \\
\text { durian }\end{array}$ & $\begin{array}{l}\text { Organic } \\
\text { fertilizer, } \\
\text { feeds }\end{array}$ & Canned milkfish, \\
\hline $\begin{array}{l}\text { Dumandan } \\
\text { Greenfields } \\
\text { Diversified } \\
\text { Farm }\end{array}$ & 2 & $\begin{array}{l}\text { Agroforestry } \\
\text { Multi-level } \\
\text { farming }\end{array}$ & & & & $\begin{array}{l}\text { Cacao, } \\
\text { lanzones }\end{array}$ & & \\
\hline $\begin{array}{l}\text { Lao } \\
\text { Integrated } \\
\text { Farm, Inc. }\end{array}$ & 5 & $\begin{array}{l}\text { Agro-forestry, } \\
\text { SALT, organic } \\
\text { farming }\end{array}$ & Goat & & & $\begin{array}{l}\text { Mangosteen, } \\
\text { durian, } \\
\text { lanzones, } \\
\text { rambutan }\end{array}$ & $\begin{array}{l}\text { Organic } \\
\text { fertilizer, } \\
\text { vermicast, } \\
\text { vermin- } \\
\text { compost }\end{array}$ & $\begin{array}{l}\text { Coconut sugar, } \\
\text { milk }\end{array}$ \\
\hline $\begin{array}{l}\text { Mindanao } \\
\text { Baptist } \\
\text { Rural Life } \\
\text { Center }\end{array}$ & 19 & $\begin{array}{l}\text { SALT, soil } \\
\text { conservation } \\
\text { technology, } \\
\text { diversified } \\
\text { farming } \\
\text { systems, } \\
\text { organic } \\
\text { farming }\end{array}$ & $\begin{array}{l}\text { Chicken } \\
\text { (layers and } \\
\text { broilers), } \\
\text { Goats, } \\
\text { Rabbits, } \\
\text { Hogs }\end{array}$ & Tilapia & $\begin{array}{l}\text { Vegetables } \\
\text { (eggplant, } \\
\text { tomato, okra, } \\
\text { squash, } \\
\text { ampalaya, } \\
\text { beans, sweet } \\
\text { pepper, } \\
\text { cucumber, } \\
\text { upo) }\end{array}$ & $\begin{array}{l}\text { Durian, } \\
\text { rambutan, } \\
\text { lanzones, } \\
\text { mangosteen, } \\
\text { calamansi } \\
\text { coffee and } \\
\text { cacao }\end{array}$ & $\begin{array}{l}\text { Fruit } \\
\text { seedlings } \\
\text { Planting } \\
\text { material } \\
\text { for } \\
\text { bamboo } \\
\text { and rattan } \\
\text { Seeds for } \\
\text { hedgerow } \\
\text { Organic } \\
\text { fertilizer } \\
\text { and } \\
\text { vermicast }\end{array}$ & Goats milk \\
\hline $\begin{array}{l}\text { Paradise } \\
\text { Organic } \\
\text { Farm }\end{array}$ & 5 & $\begin{array}{l}\text { Diversified } \\
\text { farming } \\
\text { systems, } \\
\text { organic } \\
\text { farming }\end{array}$ & & & $\begin{array}{l}\text { Vegetable; egg } \\
\text { plant, string } \\
\text { beans, okra } \\
\text { Rice }\end{array}$ & $\begin{array}{l}\text { Fruits: durian, } \\
\text { mangosteen, } \\
\text { mango }\end{array}$ & & $\begin{array}{l}\text { Herbal medicine } \\
\text { and OPV } \\
\text { vegetable seeds, } \\
\text { organic rice }\end{array}$ \\
\hline
\end{tabular}


At Paradise Organic Farm they sell organic fresh fruits, herbal medicines \& supplements, organic fertilizer, OPV vegetable seeds, organic vegetable \& rice while Dumandan Greenfields Diversified Farm The size of farms ranged from 2 hectares to 19 hectares. The major products the respondents are producing vary based on farm production specialization. Among the farm produce of ACES Natural Farm, were the following: naturally grown hog, chicken, milkfish, vegetables, organic fertilizer and feeds, canned milkfish and pork. At Binahon Agroforestry Farm they sell seedlings of various forest species such as Caribbean pine trees, lawaan, falcata, abaca, and eucalyptus trees seedling nursery, high value vegetables such as cabbage, carrots, cauliflower, lettuce, tomatoes, potato, green onions, raise farm animals such as carabao, horse, goat, sheep, hogs and other livestocks. They also grow exotic honeybees and tilapia.

Lao Integrated Farm, Inc. sells coconut sugar/syrup, fattened goats \& milk, organic fertilizer, mangosteen, durian, lanzones, rambutan, rubber, agro-forest trees. At Mindanao Baptist Rural Life Center; crops \& livestock, fruit tree seedlings (durian, rambutan, lanzones, mangosteen, calamansi, etc), plantation trees planting materials such as bamboo and rattan, vegetables seeds and products (eggplant, tomato, okra, squash, ampalaya, beans, sweet pepper, cucumber, upo), tilapia, chicken (layers and broilers), goats, rabbits, hog and seeds for hedgerows. Table 1 summarizes the technology and products of the farms.

\subsection{Attractions, Events, Recreational Activities, Educational Interventions}

The main idea of a farm tourism is to provide attractions that may or may not be directly related to farming but can promote agricultural education and relaxation. Farm tourism sites have fixed attractions, recreational activities, events, other services and educational interventions.

Fixed attractions include hostels or restaurants where people can come up and have food or conduct events such as weddings and other celebrations and social activities. Attractions also include a training center which can be used to accommodate learners and may be convertible to social halls when not in used.

Fixed attractions also include food processing facilities, work stations such as livestock operations or crop production sites, working farm structures: (such as barns), farm equipment; food processing facilities: (juice, coco sugar/syrup); recreation facilities: (camping/picnicking areas, agricultural displays) and natural area (geological features). The facilities available in the farm includes feed mill, classroom/laboratory room, vermi-beds, foliar fertilizers, organic gardens, hog houses, chicken pens, cattle condos, concoction facilities, cable way, etc., facilities and activities on fruit nursery, organic herbal garden, vam production facility, organic fertilizer production facility and herbal processing laboratory.

Events normally conducted in the farm tourism areas are the conduct of conferences, family reunions, weddings, food and craft shows, food cooking demonstrations, historic re-enactments, flower shows, corporate tours, seasonal festivals, and seasonal festivals. As such, farm tourism site who offer these events also have bed and breakfast facilities or food business operations.

Recreational activities include were water-based activity: boating, swimming, fishing activities, and chicken boxing, nature appreciation activities: photography, bird/wildlife viewing, trail based activities such as trekking, hiking, mountain biking, cycling, outdoor and "hard adventure" activities which includes rock climbing, hiking "soft adventure" activities: farm wagon rides, hunting and fishing activities.

Finally, educational interventions include training programs related to the technologies used by the farm owners and these include those on on natural farming technologies that last for 15 days entitled: organic agriculture cum livelihood training program. On-site training in vermin composting and production of organic fertilizer, techno demo for research and field trials of agricultural crops, onsite training on herbal processing, onsite training in good agricultural production, onsite training on EMO, VAM, Thrycoderma, IMO, and Korean natural farming, training in Biodynamic farming, training in massage therapy. More so, according to Engr. Apostol, the highlight of their farm products are being displayed during "Tinalak" festival of the province of Cotabato and "Hinugyaw" festival of Koronadal City. 
Table 2. Attractions, Events, Recreational Activities, Educational Interventions

\begin{tabular}{|c|c|c|c|c|c|}
\hline $\begin{array}{c}\text { Farm } \\
\text { tourism } \\
\text { operator }\end{array}$ & Fixed attractions & Events & Other services & $\begin{array}{c}\text { Recreational } \\
\text { activities }\end{array}$ & $\begin{array}{l}\text { Educational } \\
\text { interventions }\end{array}$ \\
\hline \multirow[t]{5}{*}{$\begin{array}{l}\text { Binahon } \\
\text { Agroforestry } \\
\text { Farm }\end{array}$} & Hostel & $\begin{array}{l}\text { Conferences } \\
\text { Corporate tours } \\
\text { Seasonal } \\
\text { Festivals }\end{array}$ & $\begin{array}{l}\text { Bed and } \\
\text { breakfast, Elder } \\
\text { Hostel }\end{array}$ & $\begin{array}{l}\text { Trekking, hiking, } \\
\text { mountain biking, } \\
\text { hunting }\end{array}$ & $\begin{array}{l}\text { Farm tour } \\
\text { Onsite training in good } \\
\text { agricultural production }\end{array}$ \\
\hline & Organic gardens & Family reunions & $\begin{array}{l}\text { Roadside } \\
\text { stands/markets }\end{array}$ & Camping & $\begin{array}{l}\text { Training, product } \\
\text { demonstration }\end{array}$ \\
\hline & Concoction facilities & $\begin{array}{l}\text { Food and craft } \\
\text { shows }\end{array}$ & Food service & U-pick operations & Lectures \\
\hline & $\begin{array}{l}\text { Organic fertilizer } \\
\text { Production facility }\end{array}$ & $\begin{array}{l}\text { Food cooking } \\
\text { Demonstrations }\end{array}$ & & $\begin{array}{l}\text { Photography } \\
\text { Bird/wildlife } \\
\text { viewing }\end{array}$ & $\begin{array}{l}\text { Organic agriculture } \\
\text { cum livelihood training } \\
\text { program }\end{array}$ \\
\hline & & Flower shows & & & $\begin{array}{l}\text { Techno demo for } \\
\text { research and field trials } \\
\text { of agricultural crops }\end{array}$ \\
\hline \multirow[t]{4}{*}{$\begin{array}{l}\text { ACES } \\
\text { Natural } \\
\text { Farm }\end{array}$} & Hostel & $\begin{array}{l}\text { Food and craft } \\
\text { shows }\end{array}$ & $\begin{array}{l}\text { Bed and } \\
\text { breakfast, elder } \\
\text { Hostel }\end{array}$ & Fishing & $\begin{array}{l}\text { Onsite training in good } \\
\text { agricultural production }\end{array}$ \\
\hline & Restaurant & $\begin{array}{l}\text { Food cooking } \\
\text { demonstrations }\end{array}$ & Farm shop & Fish Feeding & $\begin{array}{l}\text { Training, product } \\
\text { demonsstration }\end{array}$ \\
\hline & $\begin{array}{l}\text { Feed mill } \\
\text { Hog houses }\end{array}$ & $\begin{array}{l}\text { Corporate tours } \\
\text { Seasonal } \\
\text { festivals }\end{array}$ & & Chicken boxing & $\begin{array}{l}\text { Farm Tour } \\
\text { Organic agriculture } \\
\text { cum livelihood training } \\
\text { program }\end{array}$ \\
\hline & $\begin{array}{l}\text { Chicken pens } \\
\text { Organic fertilizer } \\
\text { Production facility }\end{array}$ & & & & \\
\hline \multirow[t]{2}{*}{$\begin{array}{l}\text { Dumandan } \\
\text { Greenfields } \\
\text { Diversified } \\
\text { Farm }\end{array}$} & $\begin{array}{l}\text { Classroom/laboratory } \\
\text { Room } \\
\text { Fruit nursery }\end{array}$ & Corporate tours & & U-pick operations & $\begin{array}{l}\text { Training on Cacao } \\
\text { production }\end{array}$ \\
\hline & $\begin{array}{l}\text { Organic fertilizer } \\
\text { Production facility }\end{array}$ & $\begin{array}{l}\text { Seasonal } \\
\text { festivals }\end{array}$ & & & $\begin{array}{l}\text { Techno demo for } \\
\text { research and field trials } \\
\text { of agricultural crops } \\
\text { Onsite training in good } \\
\text { agricultural production }\end{array}$ \\
\hline \multirow[t]{5}{*}{$\begin{array}{l}\text { Lao } \\
\text { Integrated } \\
\text { Farm, Inc. }\end{array}$} & Vermi-beds & $\begin{array}{l}\text { Food and craft } \\
\text { shows }\end{array}$ & Farm shop & Photography & $\begin{array}{l}\text { Training on coconut } \\
\text { sugar production } \\
\text { process and processing, }\end{array}$ \\
\hline & Chicken pens & $\begin{array}{l}\text { Food cooking } \\
\text { demonstrations }\end{array}$ & Tour operations & chicken boxing & $\begin{array}{l}\text { organic agriculture cum } \\
\text { livelihood training } \\
\text { program }\end{array}$ \\
\hline & Concoction facilities & $\begin{array}{l}\text { Seasonal } \\
\text { festivals }\end{array}$ & $\begin{array}{l}\text { Specialty food } \\
\text { and craft products }\end{array}$ & Trekking & $\begin{array}{l}\text { Techno demo for } \\
\text { research and field trials } \\
\text { of agricultural crops }\end{array}$ \\
\hline & Fruit nursery & & $\begin{array}{l}\text { Roadside } \\
\text { stands/markets }\end{array}$ & $\begin{array}{l}\text { Bird/wildlife } \\
\text { viewing }\end{array}$ & \\
\hline & $\begin{array}{l}\text { Organic fertilizer } \\
\text { Production facility }\end{array}$ & & $\begin{array}{l}\text { Bakeshop and } \\
\text { canteen }\end{array}$ & Hiking, biking & \\
\hline MBLRC & $\begin{array}{l}\text { Goat houses } \\
\text { Fruit nursery }\end{array}$ & $\begin{array}{l}\text { Conferences } \\
\text { Food cooking } \\
\text { demonstrations }\end{array}$ & Bed and breakfast & Photography & $\begin{array}{l}\text { Training on Farming } \\
\text { systems, crop } \\
\text { production, livestock, } \\
\text { fish and poultry, farm } \\
\text { family health, }\end{array}$ \\
\hline
\end{tabular}




\begin{tabular}{|c|c|c|c|c|c|}
\hline & & corporate tours & & & Extension and \\
\hline & $\begin{array}{l}\text { Organic fertilizer } \\
\text { production facility }\end{array}$ & $\begin{array}{l}\text { Food and craft } \\
\text { shows } \\
\text { Seasonal } \\
\text { festivals }\end{array}$ & $\begin{array}{l}\text { Food operation } \\
\text { services }\end{array}$ & Hiking, biking & $\begin{array}{l}\text { Farm tour } \\
\text { Techno demo for } \\
\text { research and field trials } \\
\text { of agricultural crops }\end{array}$ \\
\hline $\begin{array}{l}\text { Paradise } \\
\text { Organic } \\
\text { Farm }\end{array}$ & Training Center & Corporate tours & Tour Operations & Harvest festival & $\begin{array}{l}\text { Harvest festival } \\
\text { "Tinalak" festival of } \\
\text { the province of } \\
\text { Cotabato and } \\
\text { "Hinugyaw" festival of } \\
\text { Koronadal City. }\end{array}$ \\
\hline & Cattle condos & $\begin{array}{l}\text { Seasonal } \\
\text { festivals }\end{array}$ & & Hiking & Farm Demonstration \\
\hline & Fruit nursery & & & & $\begin{array}{l}\text { Organic agriculture } \\
\text { cum livelihood training } \\
\text { program }\end{array}$ \\
\hline & $\begin{array}{l}\text { Organic herbal } \\
\text { garden }\end{array}$ & & & & $\begin{array}{l}\text { Techno demo for } \\
\text { research and field trials } \\
\text { of agricultural crops }\end{array}$ \\
\hline & $\begin{array}{l}\text { Herbal processing } \\
\text { laboratory } \\
\text { Organic fertilizer } \\
\text { production facility }\end{array}$ & & & & $\begin{array}{l}\text { Onsite training on } \\
\text { herbal processing }\end{array}$ \\
\hline
\end{tabular}

\subsection{Organizational Philosophy of Farm Tourism Operator}

All the farm tourism operators believe that putting up a farm tourism site requires investment as it financial investment in putting up facilities, enhancement of the farm landscape, improving financial, entrepreneurial and more importantly agricultural knowledge and skills. While it offers an opportunity to earn more, engaging in farm tourism is both a passion and a commitment in the promotion of one's belief.

To determine the philosophy behind the farm tourism areas, owners were interviewed about why they engaged in farm tourism. Answers were then coded and results showed that there were four philosophical influences which included economic prosperity, food safety, environmental harmony, love for nature and love for agriculture.

Economic prosperity is about showcasing that farming is profitable. To do this, operators have to make sure that they develop markets for their produce. Data revealed that respondents were able to develop more than one market outlet. ACES Natural Farm operates a restaurant and sells products to schools, hotels and local markets of Panabo City and neighbouring cities and municipalities. Binahon Agroforestry Farm operates a restaurant, sells produce to schools, hotels, local market and other market outlets in various cities. Lao Integrated Farm, Inc. operates a bakery business and sells to local market in in various cities and municipalities. Mindanao Baptist Rural Life Center operates a catering business for, local and international tourist who visits the farm. While Paradise Organic Farm sells to market outlets of key cities in Mindanao, and during festivities of the town and exhibits. Dumandan Greenfields Diversified Farm sells in agricultural fair and exhibit and the local market

Economic prosperity also refers to better community earnings through provision of local employment, increased income for small entrepreneurs lining the streets leading to the farm sites and increased generation of local taxes.

The farm operators are deeply concerned in ensuring that the customers get the value for the money by providing them fresh, clean and nutritious food. For this matter, most of the farm operators practice organic farming as a way to promote food safety. Organic farming, diversified farm production and SALT are all seen 
as a way minimize negative impact on the environment.

Activities promoted in the farm tourism sites are those that promote a healthy life style such as trekking, biking, mountain biking, cycling, outdoor "hard adventure" activities which includes rock climbing, or relaxation nature appreciation activities: photography, bird/wildlife viewing, fishing and camping.

Finally, operators all believe that a farm tourism site is the best venue to promote their love of agriculture and entice the youth and other interested investors to engage in farming. All believe that it is the best way to show that farming is a respectable and profitable venture where you can derive happiness in living close to nature and be ensured of eating safe and nutritious food. It is because of this that they have tours, maintain and activities that promote love for agriculture such as participation in agricultural activities such as fishing, fruit picking, vegetable harvesting. Operators need to make sure that they are experts in the agricultural technologies and as such take all opportunities provided for by the government line agencies and the academe in terms improving their knowledge and skills, improving their facilities and accessing financial assistance and loans.

The farm operators believe to operate a farm tourism site, it is necessary to have passion for agriculture, commitment to promote common good and business acumen.

\subsection{Agricultural Education Focus}

Clearly, education of the populace is central in the philosophy of the farm tourism operators. All see it as a way to provide knowledge and skills about agriculture and rural life. This portion discusses what is normally shared to the general populace and what methods are used when sharing knowledge and skills.

Sharing of agricultural technology is central to all farm tourism operators. What is shared is also dependent on the farm tourism operation. For example, Binahon and MBLRC share the SALT technology and the component technologies. Owing to the sloping characteristics for their farm, SALT became the technology of choice as it enables them to produce products that suit their farm environment.

Binahon Farm is engaged in agro-forestry and thus promote not only SALT but component technologies that go with it such as natural vegetative strip production, apiculture, cut-flower production. As such it engages in the marketing of planting materials for timber and fruit trees as well as various crops and vegetables (cabbage, carrots, cauliflower, lettuce, tomatoes, potato, green onions) and flowers such as calla lily, baby's breath and hydrangea.

Binahon farm adopts the farming system approach as it emphasizes the need to study the landscape, contour and environmental characteristics of the farm. The farm itself serves as a technology demonstration farm where learners can view the production process or learn hands - on about it. Binahon farm has a training facility for 50 trainees and serves organically grown vegetables and fresh juices from their farm produce. he Binahon farm conducted on site training on the approaches and techniques on Soil and water conservation (SWC), Contour farming and natural vegetative strips (NVS), vermi culture and composting, biopesticides, multi-story cropping and intercropping, and Integrated pest management.

MBLRC is a pioneer in SALT which started offering the training in 1980 and by mid- 1908's it has attracted more than 1,000 learners a year. The thrust of the institution is the promotion of sustainable farming practices that can help upland farmers become more productive. Thus, central to its educational intervention is soil conservation to ensure increased productivity and income. It promotes diversified farming systems by combining trees, livestock and vegetable production and subscribes to Food Always in the Home (FAITH) gardening by demonstrating raised garden beds. The trainings offered are divided into farming systems, crop production, livestock, fish and poultry production and farm family health, extension and community development. It accommodates $20-25$ participants per training.

Lao Farm on the other hand initially worked on the existing coconut farm of his father. Having benefitted from SALT, he now expanded production of durian fruit trees and has integrated livestock in the coconut and fruit tree production. Eventually, he ventured into processing and not produces export quality products of 
coco - sugar and coco syrup and has ventured into durian candies and jam as as well as goat's milk ice cream. The technologies he shares include SALT and production of organic fertilizer.

PARADISE farm is supported and recognized in the region by Agricultural Training Institute in cultural practices of growing organic soybeans, corn, cucumber, vermin farming, herbal processing and herbal dietary supplements, raising range of native chickens, vermin productions and integrated farming systems, and therapeutic massage.

ACES is TESDA and ATI extension accredited training provider which gives training on integrated organic farming which combines fish and feeds, livestock, crops and fertilizer and biological pesticide production. The training they provide is about natural farming as well as customized training based on the needs of the client.

Dumandan Greenfield Diversified Farm is the seminar and training site in an open space surrounded by coconut trees, cacao and coffee plants, all bursting with fruits. He shared to the farmers and interested groups or individuals the importance of organic farming that works best with vegetable plants, but not quite with multiple fruit trees. Organic fertilizer (basically N-P-K, for nitrogen, phosphorus and potassium) contains mainly the three elements. Under the science of balanced plant nutrition, plants need 16 elements, hence the need to supplement with the other micro, macro and other elements.

\subsection{Approaches and Methods Used in Education}

The extension methods used by the extension providers to the farm tourism owner- operators are the following: individual (meeting and visitation), group (farm demonstration, TOT, training. The role of extension and training is crucial in the development of knowledge, perceptions and attitudes about agricultural innovations. Scherr (1992) described five basic models for extension for agroforestry practices: 'media-based extension', 'commodity-based extension', 'training and visit', 'farming systems research and extension' and 'community-based extension'. As agricultural production systems can vary considerably in nature and complexity in different settings, it is important to take these differences into account in tailoring extension interventions (Bernet et al. 2001). There has been a growing emphasis on farmer-led extension, in which farmers are the principal agents of change in their community and help disseminate the new technology to other farmers (Franzel et al. 2001, 2004, Kiptot et al. 2006. The importance of field tour, participatory discussion and exchange of ideas) and mass media (phone, email and internet) methods has been generally accepted to complement local knowledge and farmer innovation. One of the respondents on the other hand, differ among the rest of farm tourism owner-operators in terms of extension method used by the extension provider, instead they themselves provide capability building in training groups of farmers as requested by the DTI, and other agencies and organizations in the government or private sectors.

Table 3. Farm Tourism knowledge transfer approaches and methods

\begin{tabular}{lll}
\hline Farm tourism operator & Interpersonal & Mass media \\
\hline Binahon Farm & Group training & $\begin{array}{l}\text { Phone, internet, email, AVP, videos, } \\
\text { newsletters, leaflets, module materials, } \\
\text { poster, billboards }\end{array}$ \\
ACES & & $\begin{array}{l}\text { Phone, brochures, leaflets, videos, leaflets, } \\
\text { videos, module materials, posters, } \\
\text { tarpaulins, billboards }\end{array}$ \\
Natural Farm & Training/seminar & $\begin{array}{l}\text { Leaflets, tarpaulins, brochures, billboards } \\
\text { phone, email, internet, brochures, leaflets, } \\
\text { videos, newsletter, tarpaulin, billboards }\end{array}$ \\
Lamandan Greenfields Diversified Farm & Training/seminar, farm visit & $\begin{array}{l}\text { Phone, email, module materials, posters, } \\
\text { leaflets, brochures, newsletter, videos, } \\
\text { billboards }\end{array}$ \\
& meeting \& visit, training/seminar & Phone, email, leaflets, brochures, \\
nBLRC & Training/seminar, farm visit & newsletter, billboards, tarpaulins
\end{tabular}


To enhance information on farm tourism, the extension providers distributed information and communication (IEC) materials. Six (6) of the respondents express that they were able to received communication materials in terms of leaflets, videos, newsletters, module materials, posters, slides, tarpaulins, billboards, and brochures. The communication materials were provided by ATI, DA, DOST, PCAARRD and Academe. According to one respondent, the communication materials were very helpful. However, there is a need to give priority on topic related to indigenous farming system on agroforestry. Another respondent raised some clarifications on the materials, on which the season and rainfall do not present a pattern and thus, becomes a production problem. (Table 3)

\subsection{Farm Visitors}

Various kinds of people visit the farms to learn about its operations or its technologies. Most of the visitors or learners come from various government line agencies and schools who come and bring their clients. Individual enthusiasts come in third followed by private companies who come and enjoy the food and scenery of farm tourism area. Table 5 below shows the list of institutions which bring various learners to farm tourism areas.

\section{Conclusions}

Agricultural knowledge sharing among farm tourism providers in Mindanao is vibrant and reflects a promise of becoming a major factor in strengthening the economy and promoting agriculture and tourism.

1. The study revealed that farm tourism is focused on agroforestry, organic feeds and fertilizers, crops (fruit tree, vegetable, beverages, and trees), livestock, fish, and poultry production.

2. Most of the farms are diversified, produce various products and offer a variety of other service such as provision of training, catering or operation of restaurants

3. Philosophy of farm tourism operators revolve around economic, environment, health, education and have passion for agriculture, commitment to promote common good and business acumen.

4. Most of the eco-tourism areas are venues for knowledge sharing through farm visitations, tours and training programs.

5. Multiple extension methods were employed in the agricultural knowledge shared in farm tourism. These were agricultural extension participatory method training and visit approaches, and mass media method.

6. Farm tourism owner operators transfer technologies to the farmers, students, researchers, LGUs officials, participants/trainees during seminars/trainings, farm visitation, informal sharing and discussion, and technology exhibition.

7. The types of visitors dominated by trainees/participants on technology forum/training/seminar, farmers, LGUs officials, academe/school, private and international clients

8. While the farm tourism operators are constrained in terms of investment capital, the infusion of support from ATI in terms of provision of farm tourism. However, there is a need to improve the assistance of the extension providers.

\section{References}

Agunga, R., 2008. A Study of Organic Farming in Ohio with Lessons for Developing Countries.

Aquino, Marlowe U., 2008. Agritourism Development in the Philippines.

Ary, D., Jacobs, L. C., \& Razavieh, A. 2002. Introduction to Research in Education. (6th ed.) Belmont, CA: Wadsworth.

Bull, N. H., Cote, L. S., Warner, P. D., \& Mckinnie, M. R., 2004. Is Extension Relevant for the $21^{\text {st }}$ century? [Electronic version]. 
Journal of Extension, 42.

Burkhart-Kriesel, C. \& Francis, C., 2007. Red Carpet Service-linking Rural Communities to Travellers and Tourists [Electronic version]. Journal of Extension, 45.

Cromartie, J., 2007. Measuring Rurality: What Is Rural? Retrieved February 21 , 2009 from http://www.ers.usda.gov/Briefing/Rurality/WhatisRural/

Daudu, S., et.al., 2009. Agricultural Information Sources Utilized By Farmers In Benue State, Nigeria.

Dillman, D. A., 2007. Mail and Internet Surveys: The Tailored Design Method. (2nd ed.) Hoboken, NJ: John \& Wiley Sons, Inc.

Esplana, E.R., 2011. Development in the Supply Chain of the Philippine Agri-tourism Industry: An Assessment

FLORA, C. B., \& FLORA, J. L., 2008. Rural communities: legacy and change (3rd ed.). Boulder, Colorado: Westview Press.

Geisler, M., 2008. Agritourism Profile. Retrieved March 12, 2008, from http://www.agmrc.org/agmrc/commodity/agritourismprof ile.htm

Graham Busby, Samantha Rendle., 2000. The Transition from Tourism on Farms to Farm Tourism. Tourism Management 21 (2000) $635\} 642$

Iowa Department of Economic Development., 2009. Iowa tourism results. Retrieved April 14, 2009 from http://www.traveliowa.com/downloads/tourism_results.pdf

Iowa State University Extension., 2009. Visit Iowa farms. Retrieved February 2, 2009, from http://www.extension.iastate.edu/VisitIowaFarms/ Iowa State University Extension (2007).

Jensen, K., Lindborg, C., English, B., \& Menard, J., 2006. Visitors to Tennessee Agritourism Attractions: Demographics, Preferences, Expenditures, \& Projected Economic Impacts. Retrieved February 18, 2008, from University of Tennessee Web site: http://web.utk.edu/ aimag/pubs/research\%20report\%20visitors\% 20surveys3.pdf

Jolly, D. \& Reynolds, K., 2005. Consumer Demand for Agricultural and On-farm Nature Tourism. Small Farm Center, University of California-Davis.

Komar, S., 2008. New Jersey Agritourism Survey: Highlands Region. Retrieved February 4, 2008 from http://www.sussex.nj.us/Cit-eAccess/news/?TID=7\&NID=10907

Kuches, K., Toensmeyer, U. C., German, C. L., \& Bacon, J. R., 1999. An Analysis of Consumers' Views and Preferences Regarding Farmer to Consumer Direct Markets in Deleware. Retrieved January 24, 2009 from http://ageconsearch.umn.edu/ bitstream/26783/1/30010124.pdf

Kursat Demiryurek., 2008. Analysis of Information Systems and Communication Networks for Organic and Conventional Hazelnut Producers in the Samsun Province of Turkey

Leopold., 2008. Center for Sustainable Agriculture. Annual report 07-08. Ames, IA

Lobo, R. E., Goldman, G. E., Jolly, D. A., Wallace, B. D., Schrader, W. L., \& Psrker, S., 1999. Agricultural Tourism: Agritourism Benefits Agriculture in San Diego Country. Retrieved June 4, 2008, from the University of California-Davis Small Farm Center Web site: http://www.sfc.ucdavis.edu/agritourism/agritourSD.html

Mcgehehee, N. G. \& KIM, K., 2004. Motivation for agri-tourism entrepreneurship [Electronic version]. Journal of Travel Research, 43.

National Economic and Development Authority. Mindanao Strategic Development Framework, 2010-2020.

Nickerson, N. P., Black, R. J., \& Mccool, S. F., 2001. Agritourism: Motivations Behind Farm/Ranch Business Diversification. Retrieved February 8, 2008, from http://jtr.sagepub.com/cgi/content/abstract/40/1/19

Pirog, R., 2009. Local Foods: Farm Fresh and Environmentally Friendly. Retrieved March 1 , 2009 From http://www.leopold.iastate.edu/research/marketing files/ WorldBook.pdf

Putzel, S., 1984. Farm holidays combining agriculture and recreation. Agrologist, Fall: 20-21.

Schroeder, T., 2004. Motivations of Resource-Based Tourism Operators in North Dakota [Electronic version]. Journal of Extension, 42.

Seevers B., Graham D., \& Conklin, N., 2007. Education through Cooperative Extension. Columbus, OH: The Ohio State University.

Sukanlaya Choenkwan, Arunee Promkhambut, Fukui Hayao, And A. Terry Rambo., 2016. Does Agrotourism Benefit Mountain Farmers? A Case Study in Phu Ruea District, Northeast Thailand. Mountain Research and Development, 36(2):162-172. Published By: International Mountain Society

US Census Bureau., 2008. Iowa: state and county quick facts. Retrieved June 24, 2008 from http://quickfacts.census.gov/qfd/states/19000.html

US Census of Agriculture., 2007. Full 2007 census report. Retrieved February 21, 2009 from http://www.agcensus.usda.gov/Publications/2007/Full_Report/usv1.pdf 